\title{
A database of synthetic photometry in the GALEX ultraviolet bands for the stellar sources observed with the International Ultraviolet Explorer ${ }^{\star}$
}

\author{
Leire Beitia-Antero and Ana I. Gómez de Castro
}

\begin{abstract}
AEGORA Research Group, Universidad Complutense de Madrid, Facultad de CC. Matemáticas, Plaza de Ciencias 3, 28040 Madrid, Spain

e-mail: aig@ucm.es
\end{abstract}

Received 18 November 2015 / Accepted 19 September 2016

\begin{abstract}
Context. The Galaxy Evolution Explorer (GALEX) has produced the largest photometric catalogue of ultraviolet (UV) sources. As such, it has defined the new standard bands for UV photometry: the near UV band (NUV) and the far UV band (FUV). However, due to brightness limits, the GALEX mission has avoided the Galactic plane which is crucial for astrophysical research and future space missions.

Aims. The International Ultraviolet Explorer (IUE) satellite obtained 63755 spectra in the low dispersion mode $(\lambda / \delta \lambda \sim 300)$ during its 18-year lifetime. We have derived the photometry in the GALEX bands for the stellar sources in the IUE Archive to extend the GALEX database with observations including the Galactic plane.

Methods. Good quality spectra have been selected for the IUE classes of stellar sources. The GALEX FUV and NUV magnitudes have been computed using the GALEX transmission curves, as well as the conversion equations between flux and magnitudes provided by the mission.

Results. Consistency between GALEX and IUE synthetic photometries has been tested using white dwarfs (WD) contained in both samples. The non-linear response performance of GALEX inferred from this data agrees with the results from GALEX calibration. The photometric database is made available to the community through the services of the Centre de Données Stellaires at Strasbourg (CDS). The catalogue contains FUV magnitudes for 1628 sources, ranging from FUV $=1.81$ to FUV $=18.65$ mag. In the NUV band, the catalogue includes observations for 999 stars ranging from NUV $=3.34$ to NUV $=17.74$ mag.

Conclusions. UV photometry for 1490 sources not included in the GALEX AIS GR5 catalogue is provided; most of them are hot (O-A spectral type) stars. The sources in the catalogue are distributed over the full sky, including the Galactic plane.
\end{abstract}

Key words. astronomical databases: miscellaneous - catalogs - surveys - ultraviolet: stars

\section{Introduction}

The catalogue of ultraviolet (UV) sources generated by the Galaxy Evolution Explorer ${ }^{1}$ (GALEX) mission constitutes the most extensive database of UV photometry (Martin et al. 2005, hereafter Ma2005; Bianchi 2014, hereafter B2014). As such, the GALEX near ultraviolet or NUV band and far ultraviolet or FUV band, have become standards for the description of the spectral energy distribution (SED) of sources in broad band photometry. The NUV band ranges from $1771 \AA$ to $2831 \AA$, with an effective wavelength of $2315.7 \AA$, and the FUV band ranges from $1344 \AA$ to $1786 \AA$ with an effective wavelength of $1538.6 \AA$ (Morrissey et al. 2007, hereafter M2007).

The UV detectors used by the GALEX mission were sensitive Microchannel Plate (MCP) type detectors with a global count rate limit of 100000 counts s${ }^{-1}$ (M2007). As a result, the GALEX survey avoided the Galactic plane (B2014). Moreover,

* Full Tables A.1 and B.1 are only available at the CDS via anonymous ftp to cdsarc.u-strasbg. fr (130.79.128.5) or via http://cdsarc.u-strasbg.fr/viz-bin/qcat?]/A+A/596/A49 1 http://galexgi.gsfc.nasa.gov the photometric database does not extend to bright UV sources that are ideally suited for calibration purposes.

The International Ultraviolet Explorer (IUE) (Boggess et al. 1978, herefater B1978) contains the largest data set of UV spectra. Most of them were obtained in photometric conditions: good guiding, large aperture $(10 \times 20$ arcsec $)$, and low dispersion $(\lambda / \delta \lambda \sim 300)$. We have used this spectral data base to compute the FUV and NUV synthetic magnitudes of stellar sources observed with IUE under this configuration (31 982 spectra).

In this research note, we describe the methods followed to derive the FUV and NUV magnitudes from the IUE spectra, and quantify the photometric accuracy of these results by comparing GALEX and IUE-based photometry for the white dwarfs (WDs) contained in both samples. We also describe the contents and characteristics of the database available at the Centre de Données Stellaires at Strasbourg (CDS).

\section{The IUE database of stellar spectra}

The IUE database contains 63755 spectra obtained through the large aperture $(10 \times 20$ arcsec $)$ in low dispersion mode; from 
those, we have selected only the stellar sources ${ }^{2}$. This amounts to a grand total of 31982 stellar spectra ${ }^{3}$.

The IUE low dispersion spectra were recorded with three cameras: long wavelength prime (LWP), long wavelength redundant (LWR), and short wavelength prime (SWP) (B1978). From the 31982 stellar spectra, 16467 are SWP observations, 10349 are LWP observations, and 5166 are LWR observations. Good quality spectra (according to the criteria defined in Sect. 4) are available for 1889 stars in the SWP camera and 1157 stars in the LWP or LWR cameras.

\section{The GALEX photometric bands in the IUE spectra}

GALEX was a 50-cm primary space telescope with a RitcheyChrétien mounting, simultaneously feeding two detectors sensitive to near and far UV making use of a multilayer dichroic beamsplitter (Morrissey et al. 2005, Ma2005). The GALEX photometric bands are defined in the mission documentation (M2007) and can be downloaded from the GALEX official webpage $^{4}$. The FUV and NUV bands cover the spectral ranges 1344-1786 $\AA$ and 1771-2831 $\AA$ respectively (M2007); the transmittance curves are shown in Fig. 1. FUV and NUV $\mathrm{AB}$ magnitudes are determined by means of the conversion:

$$
\begin{aligned}
& \text { FUV }=-2.5 \times \log \left(\frac{\text { Flux FUV }}{1.40 \times 10^{-15} \operatorname{erg~s}^{-1} \mathrm{~cm}^{-2} \AA^{-1}}\right)+18.82 \\
& \mathrm{NUV}=-2.5 \times \log \left(\frac{\text { Flux NUV }}{2.06 \times 10^{-16} \operatorname{erg~s}^{-1} \mathrm{~cm}^{-2} \AA^{-1}}\right)+20.08
\end{aligned}
$$

\section{(M2007).}

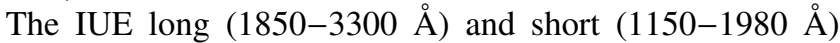
wavelength ranges (B1978) do not match exactly the GALEX bands. While GALEX FUV is completely contained in the IUE SW range, GALEX NUV is split between the LW and SW cameras. For this reason, we need to join SWP and LW spectra in order to compute NUV magnitudes. Therefore NUV photometry can only be provided for non-variable sources.

\section{Methods}

\subsection{Data selection}

Spectra have been retrieved from the IUE Newly Extracted Spectra (INES) Archive; data in this archive were processed using an optimised extraction algorithm that prevents the introduction of artefacts during the extraction while preserving the photometric accuracy (see Rodríguez-Pascual et al. 1999, for more details). The INES Archive is a final release from the European IUE Observatory.

The spectra are provided as FITS files binary tables that include four columns with: Wavelength $(\AA)$, Flux (erg cm $\left.\mathrm{cm}^{-2} \mathrm{~s}^{-1} \AA^{-1}\right)$, Flux error (erg cm $\left.\mathrm{cm}^{-2} \mathrm{~s}^{-1} \AA^{-1}\right)$ after pipeline

\footnotetext{
2 http://sdc.cab.inta-csic.es/ines/InForm. html $\{\#\}$ class

3 Not included in this census are: IUE sky images misclassified among the stellar sources or individual objects belonging to the SMC, LMC, clusters, and associations with identification not recognised by the Centre de Données Stellaires (CDS, Strasbourg).

4 http://galexgi.gsfc.nasa.gov/docs/galex/Documents/ PostLaunchResponseCurveData.html
}

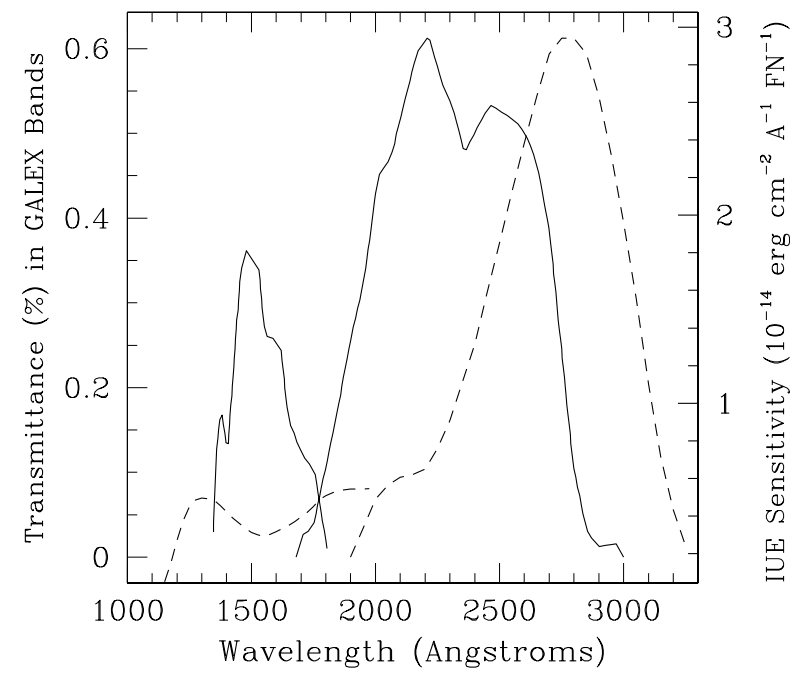

Fig. 1. GALEX transmission curves (solid) compared with IUE sensitivity curves (dashed) for the SWP and LWR cameras from Bohlin et al. (1980).

processing ${ }^{5}$, and Quality Flag (QF), a quality measure for each pixel. $Q F=0$ indicates that the pixel flux is reliable, otherwise $Q F<0$. Negative QFs are given in powers of $2\left(Q F=-2^{n}\right.$ with $n=1 \ldots 14)$ and $n$ is an identifier of the various possible sources of inaccuracies in the pixel photometry: microphonic noise, saturated pixel, reseaux mark, and others ${ }^{6}$.

The database contains all data including overexposed or underexposed spectra as well as observations that suffered problems during the downlink, calibration, processing and other processes. To avoid including bad spectra in the photometric database, we removed from our list:

- spectra with more than $10 \%$ of the pixels in the IUE spectrum flagged with $Q F<0$. The vast majority of the spectra contain reseau marks for the geometric calibration of the raw data and pixels flagged with $Q F<0$ close to the edge of the spectrum. For this reason we have worked with a tolerance of $10 \%$;

- spectra with average Flux smaller than three times the average Flux error in the corresponding GALEX wavelength range. Calibrated data include flux and flux error at each wavelength; this is the standard $3 \sigma$ criterium applied to remove underexposed spectra from the working list.

LW spectra require a more detailed examination since the peak transmittance of the GALEX NUV filter is at $2300 \AA$, in an area often underexposed in the spectrum of the cool stars observed with the IUE. To evaluate the Signal-to-Noise Ratio (SNR) in this region, we define four windows in the 1975-2375 $\AA$ range: REG I (1975-2075 $)$, REG II (2075-2175 $)$, REG III (2175-2275 $\AA$ ), and REG IV (2275-2375 $)$ ). Within these windows we compute the mean flux and the dispersion. We reject the spectra if the mean flux is negative or if the standard deviation is ten times higher than the mean in any of the regions II-III-IV. We allow for a factor of ten to prevent removing sources with steep energy distributions or strong features.

5 See Gonzalez-Riestra et al. (2001) for the absolute calibration of the INES IUE fluxes.

6 See https://archive.stci.eduiue/manual/newsips/ node20. html for details. 


\subsection{Calculation of the FUV and NUV synthetic magnitudes}

The integrated flux in the FUV band is computed from the SWP IUE spectra after multiplication by the normalised transmittance of the FUV filter. The flux of the flagged bad pixels $(Q F<0)$ has been interpolated from good nearby pixels. The $\mathrm{AB}$ magnitude is calculated using Eq. (1) (see Sect. 3).

To evaluate the NUV synthetic magnitude, it is first necessary to determine whether the sources are variable (see Sect. 4.4 for a description of the procedure). In case there is no evidence of variability, the SW and LW are joined into a single spectrum; the matching wavelength is set at $1975 \AA$. After this, the spectrum is multiplied by the normalised transmittance of the NUV filter. The flux of the flagged bad pixels $(Q F<0)$ has been interpolated from good nearby pixels. The $\mathrm{AB}$ magnitude is calculated using Eq. (2) (see Sect. 3). In case there are multiple observations and the source is found not to vary, synthetic magnitudes are computed from the average flux.

\subsection{Error determination}

For each spectrum and band, the Flux error provided by the mission (Col. \#3 in the data) is multiplied by the GALEX normalised transmittance to evaluate the total error in each band denoted as $S_{\mathrm{FUV}}$ and $S_{\mathrm{NUV}}$ for the FUV and NUV bands respectively. From that, magnitude errors are provided for the FUV band, eFUV ${ }^{+}$, and $\mathrm{eFUV}^{-}$, and NUV band, $\mathrm{eNUV}^{+}$, and $\mathrm{eNUV}^{-}$; the errors are asymmetric around the magnitude value because of the logarithmic scale. In case there are multiple observations and the source is found not to vary, errors are from the Flux errors.

\subsection{Multiple observations. Variable stars}

There are 598 stars in the IUE archive with multiple observations in the SW range and 409 stars with multiple observations in the LW range. For those, variability has been tested.

\subsubsection{Stars with multiple observations in the IUE SW range}

We compute for each SWP observation, $i$, the weighted flux in the GALEX FUV band, $F_{\mathrm{FUV}}(i)$, calculated as:

$F_{\mathrm{FUV}}(i)=\int F_{i}(\lambda) G_{\mathrm{FUV}}(\lambda) \mathrm{d} \lambda$,

with $G_{\mathrm{FUV}}$ being the normalised GALEX FUV transmission curve (see Fig. 1). The weighted Flux error, $S_{\mathrm{FUV}}(\mathrm{i})$, is determined in the same manner:

$S_{\mathrm{FUV}}(i)=\int S_{i}(\lambda) G_{\mathrm{FUV}}(\lambda) \mathrm{d} \lambda$,

with $S_{\text {FUV }}(i)$ being the Flux error (see Fig. 1).

After this, the average $\left\langle F_{\mathrm{FUV}}\right\rangle$, the dispersion $\sigma\left(F_{\mathrm{FUV}}\right)$, and the average Flux error $\left\langle S_{\mathrm{FUV}}\right\rangle$ are computed. We flag a star as variable if $\sigma\left(F_{\mathrm{FUV}}\right) \geq 3 \times\left\langle S_{\mathrm{FUV}}\right\rangle$. Very noisy data have already been rejected in the data selection process (see Sect. 4.1).

\subsubsection{Stars with multiple observations in the IUE LW range}

The procedure is similar to that described for the SW range but, in this case, the variability test is carried out only over the range of the NUV band contained in the LW images.
We compute for each LW observation, $i$, the weighted flux in the 1975-3000 $\AA$ range, $F_{\mathrm{GaLW}}(i)$, calculated as:

$F_{\mathrm{GaLW}}(i)=\int_{1975}^{3000} F_{i}(\lambda) G_{\mathrm{NUV}}(\lambda) \mathrm{d} \lambda$,

with $G_{\mathrm{NUV}}$ being the normalised GALEX NUV transmission curve (see Fig. 1), as well as the weighted Flux error, $S_{\text {GaLW }}(\mathrm{i})$,

$S_{\mathrm{GaLW}}(i)=\int_{1975}^{3000} S_{i}(\lambda) G_{\mathrm{NUV}}(\lambda) \mathrm{d} \lambda$.

The average $\left\langle F_{\mathrm{GaLW}}\right\rangle$, the dispersion $\sigma\left(F_{\mathrm{GaLW}}\right)$, and the average Flux error $\left\langle S_{\mathrm{GaLW}}\right\rangle$ are then computed. We flag a star as variable if $\sigma\left(F_{\mathrm{GaLW}}\right) \geq 3 \times\left\langle S_{\mathrm{GaLW}}\right\rangle$.

\subsubsection{In summary}

According to these criteria, 52 stars are found to be variable in the LW range and 88 in the SW range; only 36 stars are found to be variable in both ranges. Therefore, $\sim 13 \%$ of the stars with multiple observations in the LW range are found to be variable and $\sim 15 \%$ of the stars with multiple observations in the SW range are found to be variable.

An additional search for variable sources was carried out by comparing the fluxes in the window where SW and LW spectra overlap for every source. Three additional stars have been identified as variables from this test, namely HD 37209, HD 56014, and HD 14399, and have been excluded from the catalogue.

\section{Photometric accuracy}

The IUE sample (of non-variable stars) provides good synthetic photometry for 103 WDs. 43 of these 103 WDs have a counterpart in the GALEX GR5 AIS survey (Bianchi et al. 2011) within a search radius of $3^{\prime \prime}$. We have used this subset to check the photometric accuracy of the synthetic magnitudes computed in this work (see Table 1 for their synthetic and GALEX magnitudes). The limiting magnitude of GALEX AIS $^{7}$ (NUV $\sim 20.5 \mathrm{mag}$ ) is high above the sensitivity threshold of IUE in low dispersion mode; therefore counterparts are identified for all sources within the area mapped by GALEX. We note that in May 2009, the FUV detector in GALEX stopped working and as a result, releases later to GR5 (such as GR6/7) only add new sources in the NUV band ${ }^{8}$ (B2014).

As shown in Fig. 2, GALEX- and IUE-based UV photometries compare well except for the brightest sources; GALEX photometry is affected by photon count loses at high count rates. Following Camarota \& Holberg (2014, hereafter CH), we have fitted the samples to a quadratic function using the least-squares method:

$M_{\mathrm{GAL}}=c_{0}+c_{1} M_{\mathrm{IUE}}+c_{2} M_{\mathrm{IUE}}^{2}$

with $M_{\mathrm{GAL}}$ the WD magnitude as per the GALEX AIS catalogue and $M_{\text {IUE }}$ the IUE-based synthetic magnitude derived in this work. The coefficients of the fit, $c_{0}, c_{1}$, and $c_{2}$, are given in Table 2. We found a very good agreement with $\mathrm{CH}$ 's fits in the FUV photometry and a significant discrepancy in the NUV band that we ascribe to a possible typographic error in the parameters in CH's Table 2. The FUV and NUV synthetic photometry for the rest of the WDs in the IUE sample is provided in Table 3.

\footnotetext{
7 https://archive.stsci.edu/prepds/gcat/gcat_gasc_ gmsc.html

8 In fact, late GALEX releases also add very weak FUV sources, identified by being the counterpart of a NUV source. These sources are too
} weak to have been observed with IUE. 
Table 1. Synthetic photometry in the GALEX bands of WD observed with IUE: WDs with GALEX counterpart.

\begin{tabular}{|c|c|c|c|c|c|c|c|c|c|c|c|c|}
\hline ID IUE & RAS (ICRS) & Dec (ICRS) & & IUE-ba & ased synt & hetic $\mathrm{p}$ & hotometry & & & $\operatorname{ALEX}_{1}$ & hotomet & try \\
\hline & & & FUV & $+\mathrm{eFUV}$ & -eFUV & NUV & $+\mathrm{eN}$ & $-\mathrm{eN}$ & FUV & eFUV & NUV & eNUV \\
\hline WD $0005+511$ & 00:08:18.17 & $+51: 23: 16.6$ & 11.09 & 0.06 & -0.06 & NA & NA & $\mathrm{NA}$ & 12.190 & 0.004 & 13.178 & 0.004 \\
\hline EQ $0027+259$ & 00:29:37.96 & $+26: 10: 28.47$ & 13.60 & 0.07 & -0.06 & 13.22 & 0.30 & -0.24 & 13.567 & 0.008 & 13.785 & 0.006 \\
\hline WD $0039+04$ & $00: 42: 6.12$ & $+05: 09: 23.36$ & 12.01 & 0.08 & -0.07 & NA & NA & NA & NA & NA & 13.189 & 0.004 \\
\hline WD 0050-332 & $00: 53: 17.44$ & $-32: 59: 56.6$ & 11.47 & 0.07 & -0.07 & 12.13 & 0.11 & -0.10 & NA & NA & 12.365 & 0.003 \\
\hline WD $0112+104$ & $01: 14: 37.8$ & $+10: 41: 6$ & 14.40 & 0.15 & -0.13 & 14.66 & 0.28 & -0.22 & 14.425 & 0.010 & 14.623 & 0.007 \\
\hline WD $0134+833$ & 01:41:28.74 & $+83: 34: 58.9$ & 12.34 & 0.07 & -0.06 & 12.70 & 0.10 & -0.09 & 12.923 & 0.002 & 12.938 & 0.002 \\
\hline WD $0232+035$ & $02: 35: 7.59$ & $+03: 43: 56.8$ & 10.39 & 0.07 & -0.06 & & 0.11 & -0.10 & NA & NA & 12.371 & 0.003 \\
\hline WD $0302+027$ & 03:04:37.34 & $+02: 56: 57.9$ & 13.31 & 0.11 & -0.10 & 13.69 & 0.13 & -0.11 & 13.008 & 0.007 & 13.968 & 0.007 \\
\hline WD 0320-539 & 03:22:14.83 & $-53: 45: 16.5$ & 13.13 & 0.09 & -0.08 & NA & NA & NA & 13.325 & 0.004 & 13.747 & 0.003 \\
\hline WD $0342+026$ & $03: 45: 34.58$ & $+02: 47: 52.81$ & 10.44 & 0.07 & -0.07 & 10.76 & 0.12 & -0.11 & NA & NA & 12.751 & 0.003 \\
\hline WD 0453-296 & 04:55:35.94 & $-29: 28: 59.96$ & 15.01 & 0.21 & -0.17 & 15.03 & 0.23 & -0.19 & 15.029 & 0.015 & 14.995 & 0.010 \\
\hline HS $0713+3958$ & $07: 17: 2.7$ & $+39: 53: 25$ & 14.57 & 0.11 & -0.10 & 15.05 & 0.23 & -0.19 & 14.643 & 0.012 & 77 & 0.009 \\
\hline WD $0846+249$ & 08:49:5.88 & $+24: 45: 7.93$ & 14.45 & 0.08 & -0.08 & NA & NA & NA & 14.436 & 0.013 & 15.147 & 0.011 \\
\hline WD $0853+163$ & 08:56:18.96 & $+16: 11: 3.8$ & 15.28 & 0.14 & -0.13 & 15.31 & 0.50 & -0.34 & 15.273 & 0.022 & 15.201 & 0.013 \\
\hline WD $0945+246$ & 09:48:46.65 & $+24: 21: 26$ & 14.38 & 0.08 & -0.08 & 13 & 0.21 & -0.18 & 14.479 & 0.011 & 13 & 0.005 \\
\hline PG 0958-073 & 10:00:47.25 & $-07: 33: 31.01$ & 12.44 & 0.07 & -0.06 & 12.90 & 0.13 & -0.11 & 12.517 & 0.006 & 13.190 & 0.005 \\
\hline WD 1034+001 & $10: 37: 3.81$ & $-00: 08: 19.31$ & 10.85 & 0.06 & -0.06 & & 0.09 & -0.09 & NA & NA & 65 & 0.002 \\
\hline WD $1114+072$ & $11: 16: 49.35$ & $+06:$ & 11.85 & 0.07 & -0.06 & 12.24 & 0.13 & -0.11 & 12.360 & 0.003 & 13.119 & 0.004 \\
\hline WD $1144+005$ & $11: 46: 35.17$ & $+00: 12: 33.6$ & 13.69 & 0.09 & -0.09 & NA & NA & NA & 12.921 & 0.008 & 13.691 & 0.006 \\
\hline WD $1211+332$ & $12: 13: 56.25$ & $+32: 56: 31.4$ & 12.64 & 0.07 & -0.06 & 13.22 & 0.11 & -0.10 & 12.718 & 0.008 & 13.396 & 0.004 \\
\hline WD $1230+052$ & $12: 33: 12.57$ & $+04: 57: 37.7$ & 12.04 & 0.07 & -0.06 & 12.34 & 0.10 & -0.09 & 12.544 & 0.004 & 13.732 & 0.004 \\
\hline WD $1233+427$ & $12: 35: 51.14$ & $+42: 22: 39.72$ & 10.82 & 0.06 & -0.06 & & & -0.11 & 12.710 & 0.007 & 12 & 0.004 \\
\hline WD $1302+283$ & $13: 04: 48.63$ & $+28: 07: 29.3$ & 13.69 & 0.11 & -0.10 & 14.10 & 0.24 & -0.19 & 13.802 & 0.009 & 14.238 & 0.005 \\
\hline WD 1326-037 & $13: 29: 16.39$ & $-03: 58: 51.75$ & 15.22 & 0.12 & -0.11 & 15.20 & 0.33 & -0.26 & 15.295 & 0.015 & 15.204 & 0.009 \\
\hline WD $1406+590$ & $14: 08: 32.23$ & $+59: 40: 25.1$ & & 0.11 & -0.10 & & 0. & & 12.690 & 0.003 & 12.548 & 0.002 \\
\hline WD $1445+152$ & $14: 48: 14.38$ & $+15: 04: 49.93$ & 15.62 & 0.29 & -0.23 & 15.57 & 0.38 & -0.28 & 15.462 & 0.027 & 15.421 & 0.016 \\
\hline WD $1636+351$ & $16: 38: 26.32$ & $+35: 00: 11.9$ & & 0.09 & -0.09 & 13.75 & 0. & & 13.236 & 0.005 & 13 & 0.003 \\
\hline PG $1637+346$ & $16: 39: 36.02$ & $+34: 32: 30.36$ & 15.01 & 0.18 & -0.16 & NA & NA & $\mathrm{NA}$ & 14.403 & 0.008 & 14.656 & 0.005 \\
\hline HS $1650+7229$ & $16: 49: 16.1$ & $+72: 24: 12$ & 15.44 & 0.12 & -0.11 & NA & NA & NA & 15.581 & 0.032 & 16.050 & 0.013 \\
\hline WD $1708+602$ & 17:09:15.9 & $+60: 10: 11$ & 11.82 & 0.04 & -0.04 & NA & NA & $\mathrm{NA}$ & 12.707 & 0.005 & 12.565 & 0.003 \\
\hline WD 2034-532 & $20: 38: 16.84$ & $-53: 04: 25.4$ & 14.56 & 0.12 & -0.11 & NA & NA & NA & 14.533 & 0.014 & 14.190 & 0.007 \\
\hline WD 2059+013 & $21: 02: 19.3$ & $+01: 32: 15.9$ & 13.93 & 0.09 & -0.08 & 14.21 & 0.23 & -0.19 & 13.982 & 0.007 & 14.364 & 0.005 \\
\hline WD $2110+127$ & $21: 13: 21.06$ & $+12: 57: 9.4$ & 12.41 & 0.12 & -0.11 & NA & NA & NA & 12.787 & 0.006 & 13.182 & 0.005 \\
\hline BPS CS 22951- & 21:49:38.91 & $-43: 06: 14.28$ & 14.70 & 0.08 & -0.08 & 16.35 & 0.53 & -0.36 & 13.511 & 0.010 & 14.312 & 0.004 \\
\hline WD $2149+021$ & 21:52:25.38 & $+02: 23: 19.54$ & 12.24 & 0.06 & -0.06 & 12.51 & 0.12 & -0.11 & 12.604 & 0.007 & 12.688 & 0.005 \\
\hline WD 2207-303 & $22: 10: 29.22$ & $-30: 05: 43.7$ & 12.63 & 0.06 & -0.06 & NA & $\mathrm{NA}$ & NA & 12.596 & 0.004 & 13.539 & 0.003 \\
\hline HS $2237+8154$ & $22: 37: 15.57$ & $+82: 10: 27.32$ & 17.45 & 1.40 & -0.59 & NA & NA & NA & 17.396 & 0.040 & 16.923 & 0.021 \\
\hline WD $2246+223$ & $22: 49: 5.76$ & $+22: 36: 32.31$ & 17.57 & 1.27 & -0.57 & NA & NA & NA & 17.736 & 0.038 & 15.109 & 0.006 \\
\hline WD $2316+123$ & $23: 18: 45.1$ & $+12: 36: 2.9$ & 16.70 & 0.56 & -0.37 & 15.74 & 0.26 & -0.21 & 16.889 & 0.045 & 15.783 & 0.011 \\
\hline WD 2331-475 & $23: 34: 2.2$ & $-47: 14: 26.5$ & 12.17 & 0.08 & -0.07 & NA & NA & NA & 12.419 & 0.004 & 13.405 & 0.003 \\
\hline WD 2333-002 & $23: 35: 41.47$ & $+00: 02: 19$ & 14.22 & 0.12 & -0.11 & NA & NA & $\mathrm{NA}$ & 14.149 & 0.014 & 14.719 & 0.010 \\
\hline WD $2342+806$ & $23: 45: 2.26$ & $+80: 56: 59.7$ & 12. & 0.07 & -0.07 & 13.27 & 0.11 & -0.10 & 12.954 & 0.005 & 13.520 & 0.004 \\
\hline WD $2353+026$ & $23: 56: 27.72$ & $+02: 57: 5.61$ & 13.85 & 0.11 & -0.10 & 14.58 & 0.24 & -0.20 & 13.829 & 0.007 & 14.357 & 0.006 \\
\hline
\end{tabular}

Notes. NA means no available data. 

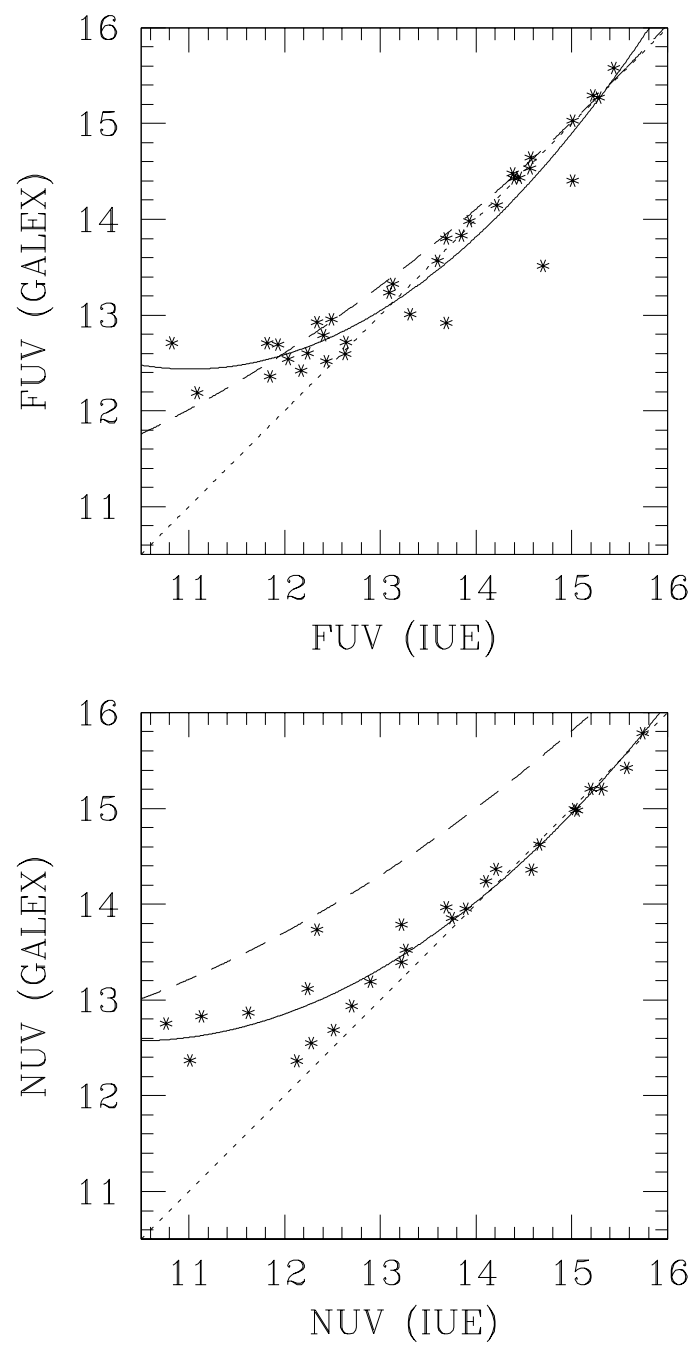

Fig. 2. IUE versus GALEX photometry for the WDs sample. The dotted line represents the 1:1 correspondence, the solid line represents the fit in Table 2 and the dashed line represents CH's fit.

\section{The catalogue}

The catalogue contains FUV magnitudes for all stars (with and without multiple observations) and NUV magnitudes only for non-variable stars, or stars with just one good observation, as pointed out in Sect. 4.2. The number of observations used for the variability evaluation are indicated for all catalogue entries; $\sim 65 \%$ of the sources have only one good observation.

Appendix A contains an excerpt of the catalogue available at the CDS. For each source, the following entries are provided:

- Object identification in the IUE Archive.

- Right Ascension and Declination (ICRS).

- Number of SWP observations used to compute FUV.

- Synthetic FUV magnitude (Eq. (4)) computed from the mean flux, if several observations are used (see Sect. 4.2).

- Error in the synthetic FUV magnitude computed from the mean flux and error (see Sect. 4.3).

- Number of LW observations used to compute NUV.

- Synthetic NUV magnitude (obtained from SWP and LW mean fluxes).

- Error in the synthetic NUV magnitude (obtained from SWP and LW mean fluxes).
Table 2. Quadratic fit parameters for the WDs sample.

\begin{tabular}{lll}
\hline \hline Property & FUV & FUV \\
\hline$c_{0}$ & 31.2738 & 24.9204 \\
$c_{1}$ & -3.4197 & -2.3688 \\
$c_{2}$ & 0.1552 & 0.1136 \\
$\chi^{2} /$ d.o.f. & 0.0064 & 0.0058 \\
No. of stars & 34 & 24 \\
Lower bound & 10.8 & 10.8 \\
Upper bound & 15.5 & 15.7 \\
\hline
\end{tabular}

For the 88 stars found to be variable in the SW range, additional entries are provided in a supplementary catalogue (an excerpt is shown in Appendix B) with the FUV synthetic magnitude for each observation (see Sect. 4.4). The following entries are provided:

- Object identification in the IUE Archive.

- Right Ascension and Declination (ICRS).

- Observation date and time.

- Synthetic FUV magnitude computed from the flux.

- Error in the synthetic FUV magnitude computed from the flux and error.

The catalogue contains synthetic FUV magnitudes for 1628 sources, ranging from FUV $=1.81$ to $\mathrm{FUV}=18.65$. In the NUV band, the catalogue includes observations for 999 stars ranging from NUV $=3.34$ to $\mathrm{NUV}=17.74 \mathrm{mag}$. The distribution of sources in the sky is plotted in Fig. 3; we highlight the good coverage of the Galactic plane. A summary statistics of the catalogue contents is available in Table 4. This work adds UV photometry for 1490 new sources, most of them hot (O-A spectral type) stars. The catalogue is available to the community through the services of the Centre de Données Stellaires.

\section{Conclusions}

From an initial sample of 31982 stellar IUE spectra, we computed the synthetic photometry for:

- 1628 sources in the GALEX FUV band with magnitudes ranging from $\mathrm{FUV}=1.81$ to $\mathrm{FUV}=18.65$.

- 999 sources in the GALEX NUV band with NUV ranging from $\mathrm{NUV}=3.34$ to NUV $=17.74 \mathrm{mag}$.

The FUV and NUV synthetic photometry compares well with GALEX. A sample of WD's observed with IUE and GALEX were used for the test; a good agreement with $\mathrm{CH}$ was found for the FUV band but not for the NUV band.

The catalogue is available to the community through the services of the Centre de Données Stellaires. It adds UV photometry for 1490 new sources with respect to the GALEX AIS catalogue, most of them hot (O-A spectral type) stars. The sources in the catalogue are distributed over the full sky, including the Galactic plane.

Acknowledgements. We want to thank the referee for his/her comments which improved this paper. this work has been supported by the Ministry of Economy and Competitiveness of Spain through grants: AYA2011-29754-c3-01, ESP2014-54243-R. Leire Beitia-Antero acknowledges the receipt of a "Beca de Colaboración" from the Ministry of Education of Spain. 
Table 3. Synthetic photometry in the GALEX bands of WDs observed with IUE: that is, WDs without GALEX counterpart.

\begin{tabular}{|c|c|c|c|c|c|c|c|c|}
\hline \multirow[t]{2}{*}{ ID IUE } & \multirow[t]{2}{*}{ RAS (ICRS) } & \multirow[t]{2}{*}{ Dec (ICRS) } & \multicolumn{6}{|c|}{ IUE-based synthetic photometry } \\
\hline & & & FUV & $+\mathrm{eFUV}$ & -eFUV & NUV & +eNUV & -eNUV \\
\hline WD $2357+296$ & $00: 00: 7.25$ & $+29: 57: 0.31$ & 13.06 & 0.11 & -0.10 & NA & NA & NA \\
\hline WD $0017+136$ & $00: 20: 1.79$ & $+13: 52: 48.1$ & 15.27 & 0.12 & -0.10 & 15.09 & 0.17 & -0.14 \\
\hline WD 0101+039 & 01:04:21.68 & $+04: 13: 37.06$ & 10.89 & 0.11 & -0.10 & 11.23 & 0.12 & -0.10 \\
\hline WD 0104+50 & 01:07:11.02 & $+51: 10: 22.74$ & 11.85 & 0.06 & -0.06 & NA & NA & NA \\
\hline WD 0128-387 & $01: 30: 28.03$ & $-38: 30: 38.7$ & 15.84 & 0.26 & -0.21 & 15.37 & 0.16 & -0.14 \\
\hline WD 0131-164 & $01: 34: 24.07$ & $-16: 07: 8.38$ & 11.98 & 0.06 & -0.06 & 12.83 & 0.11 & -0.10 \\
\hline WD 0135-052 & $01: 37: 59.34$ & $-04: 59: 44.3$ & NA & NA & NA & 15.15 & 0.26 & -0.21 \\
\hline WD 0141-675 & 01:43:1.04 & $-67: 18: 29.37$ & NA & NA & NA & 17.13 & 3.06 & -0.72 \\
\hline WD $0148+467$ & 01:52:2.96 & $+47: 00: 6.65$ & 12.60 & 0.06 & -0.05 & 12.67 & 0.14 & -0.12 \\
\hline WD 0214+568 & $02: 17: 33.52$ & $+57: 06: 47.5$ & 12.49 & 0.07 & -0.06 & NA & NA & NA \\
\hline PG $0216+032$ & $02: 19: 19$ & $+03: 26: 54$ & 12.45 & 0.06 & -0.05 & 13.18 & 0.13 & -0.11 \\
\hline WD $0227+050$ & $02: 30: 16.62$ & $+05: 15: 50.68$ & 12.10 & 0.06 & -0.05 & 12.43 & 0.10 & -0.09 \\
\hline WD 0229-481 & $02: 30: 53.3$ & $-47: 55: 26.2$ & 12.41 & 0.06 & -0.06 & NA & NA & NA \\
\hline WD $0232+525$ & $02: 36: 19.54$ & $+52: 44: 12.5$ & 13.33 & 0.07 & -0.06 & NA & NA & NA \\
\hline KUV 02503-0238 & $02: 52: 51$ & $-02: 25: 17.99$ & 14.63 & 0.12 & -0.11 & 14.76 & 0.52 & -0.35 \\
\hline WD 0255-705 & $02: 56: 17.21$ & $-70: 22: 10.86$ & NA & NA & NA & 14.84 & 1.08 & -0.53 \\
\hline WD 0346-011 & $03: 48: 50.19$ & $-00: 58: 32.02$ & 12.02 & 0.11 & -0.10 & 12.73 & 0.09 & -0.08 \\
\hline KUV 685-13 & $04: 50: 13.52$ & $+17: 42: 6.21$ & 12.07 & 0.09 & -0.08 & 12.59 & 0.16 & -0.14 \\
\hline WD 0455-282 & $04: 57: 13.9$ & $-28: 07: 54$ & 11.76 & 0.07 & -0.06 & 12.41 & 0.17 & -0.15 \\
\hline WD 0509-007 & $05: 12: 6.39$ & $-00: 42: 6$ & 12.01 & 0.06 & -0.06 & NA & NA & NA \\
\hline WD 0531-022 & $05: 34: 18$ & $-02: 15: 0$ & 14.28 & 0.43 & -0.31 & NA & NA & NA \\
\hline WD 0612+177 & $06: 15: 18.69$ & $+17: 43: 41$ & 11.93 & 0.07 & -0.07 & 12.48 & 0.17 & -0.15 \\
\hline WD $0640+015$ & $06: 43: 16.02$ & $+01: 30: 12.6$ & 13.81 & 0.08 & -0.08 & $\mathrm{NA}$ & $\mathrm{NA}$ & NA \\
\hline WD 0651-020 & $06: 54: 13$ & $-02: 09: 12$ & 12.94 & 0.07 & -0.06 & 13.59 & 0.21 & -0.17 \\
\hline WD 0715-703 & $07: 15: 16.59$ & $-70: 25: 5.6$ & 12.08 & 0.07 & -0.06 & NA & NA & NA \\
\hline WD $0836+237$ & $08: 39: 33.3$ & $+23: 34: 9$ & 14.43 & 0.45 & -0.32 & NA & NA & NA \\
\hline $\mathrm{BD}+481777$ & $09: 30: 46.78$ & $+48: 16: 23.77$ & 8.87 & 0.05 & -0.05 & 9.29 & 0.12 & -0.11 \\
\hline PG $0934+553$ & $09: 38: 20.35$ & $+55: 05: 50.08$ & 10.47 & 0.07 & -0.07 & NA & NA & NA \\
\hline WD $1013-050$ & $10: 16: 28.68$ & $-05: 20: 32.06$ & 12.07 & 0.06 & -0.06 & 12.71 & 0.11 & -0.10 \\
\hline WD $1042-690$ & $10: 44: 10.23$ & $-69: 18: 18.03$ & 11.88 & 0.05 & -0.05 & 12.29 & 0.10 & -0.09 \\
\hline WD $1105-048$ & $11: 07: 59.95$ & $-05: 09: 25.89$ & 12.90 & 0.06 & -0.05 & 13.08 & 0.10 & -0.10 \\
\hline WD $1123+189$ & $11: 26: 19.06$ & $+18: 39: 17.85$ & 12.08 & 0.06 & -0.05 & 12.73 & 0.10 & -0.09 \\
\hline WD $1234+482$ & $12: 36: 45.18$ & $+47: 55: 22.34$ & 12.30 & 0.09 & -0.08 & 12.94 & 0.11 & -0.10 \\
\hline WD $1302+597$ & $13: 04: 32.19$ & $+59: 27: 32.78$ & 13.33 & 0.07 & -0.07 & 13.66 & 0.16 & -0.14 \\
\hline WD $1321+36$ & $13: 23: 35.26$ & $+36: 07: 59.51$ & 10.11 & 0.05 & -0.05 & 10.40 & 0.10 & -0.09 \\
\hline BD-07 3632 & $13: 30: 13.64$ & $-08: 34: 29.5$ & 12.33 & 0.10 & -0.09 & 12.40 & 0.18 & -0.15 \\
\hline WD $1337+705$ & $13: 38: 50.48$ & $+70: 17: 7.66$ & 11.84 & 0.06 & -0.05 & 12.23 & 0.12 & -0.11 \\
\hline WD 1403-077 & $14: 06: 4.83$ & $-07: 58: 31.21$ & 13.66 & 0.08 & -0.08 & NA & NA & NA \\
\hline WD $1424+534$ & $14: 25: 55.46$ & $+53: 15: 25.14$ & 13.50 & 0.07 & -0.07 & 14.23 & 0.21 & -0.18 \\
\hline CD-37 10500B & $15: 47: 30.07$ & $-37: 55: 8.11$ & 16.76 & 1.73 & -0.64 & 13.97 & 0.20 & -0.17 \\
\hline WD $1615-154$ & $16: 17: 55.26$ & $-15: 35: 51.93$ & 11.65 & 0.08 & -0.08 & 12.28 & 0.11 & -0.10 \\
\hline CD-38 10980 & $16: 23: 33.84$ & $-39: 13: 46.16$ & 9.62 & 0.06 & -0.06 & 10.15 & 0.10 & -0.09 \\
\hline WD 1639+537 & $16: 40: 57.16$ & $+53: 41: 9.6$ & NA & NA & NA & 17.09 & 0.86 & -0.48 \\
\hline WD $1657+343$ & $16: 58: 51.12$ & $+34: 18: 53.29$ & 14.37 & 0.10 & -0.09 & NA & NA & NA \\
\hline WD $1713+695$ & $17: 13: 6.12$ & $+69: 31: 25.7$ & 13.07 & 0.15 & -0.13 & 13.13 & 0.51 & -0.34 \\
\hline WD $1725+586$ & $17: 26: 43.36$ & $+58: 37: 32.06$ & 13.41 & 0.25 & -0.20 & NA & NA & NA \\
\hline WD 1900+706 & 19:00:10.25 & $+70: 39: 51.2$ & 13.88 & 0.15 & -0.13 & NA & NA & NA \\
\hline ** LDS 678A & $19: 20: 34.92$ & $-07: 40: 0.07$ & 14.83 & 0.23 & -0.19 & NA & NA & NA \\
\hline WD 1936+327 & $19: 38: 28.21$ & $+32: 53: 19.9$ & 12.54 & 0.08 & -0.08 & NA & NA & NA \\
\hline LS II +189 & $19: 43: 31.21$ & $+18: 24: 34.58$ & 10.20 & 0.07 & -0.06 & 10.77 & 0.10 & -0.09 \\
\hline WD 2007-303 & $20: 10: 56.85$ & $-30: 13: 6.64$ & 12.13 & 0.06 & -0.06 & 12.28 & 0.11 & -0.10 \\
\hline RX J2013.1+4002 & $20: 13: 9.37$ & $+40: 02: 24.25$ & 12.10 & 0.06 & -0.06 & NA & NA & NA \\
\hline WD 2028+390 & $20: 29: 56.16$ & $+39: 13: 32$ & 12.02 & 0.06 & -0.06 & 12.56 & 0.17 & -0.14 \\
\hline WD 2123-82 & $21: 31: 5.18$ & $-82: 40: 53.25$ & 12.46 & 0.07 & -0.06 & 12.35 & 0.20 & -0.17 \\
\hline WD 2211-495 & $22: 14: 11.91$ & $-49: 19: 27.26$ & 9.40 & 0.05 & -0.05 & 10.23 & 0.10 & -0.09 \\
\hline WD 2313-021 & $23: 16: 12.42$ & $-01: 50: 35.06$ & 11.79 & 0.07 & -0.06 & 12.09 & 0.10 & -0.09 \\
\hline GD 1110 & $23: 19: 24.43$ & $-08: 52: 37.91$ & 11.87 & 0.06 & -0.05 & NA & NA & NA \\
\hline WD 2317-054 & $23: 19: 58.4$ & $-05: 09: 56.16$ & 10.07 & 0.05 & -0.05 & 10.47 & 0.10 & -0.09 \\
\hline GD 1309 & $23: 29: 12$ & $-10: 05: 0$ & 11.68 & 0.07 & -0.06 & 12.09 & 0.11 & -0.10 \\
\hline WD $2349+286$ & $23: 51: 56$ & $+28: 55: 12$ & 14.39 & 0.22 & -0.18 & NA & NA & NA \\
\hline
\end{tabular}

Notes. NA means no available data. 
L. Beitia-Antero and A. I. Gómez de Castro: UV photometric database

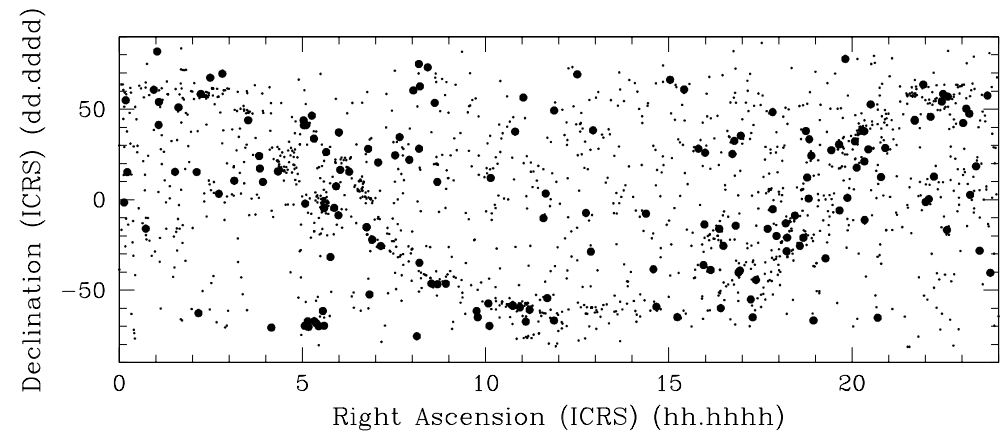

Fig. 3. Distribution in the sky of the sources in the catalogue. Variable sources (as per the criteria in Sect. 4.4) are indicated with filled circles.

Table 4. Catalogue contents.

\begin{tabular}{|c|c|c|c|c|c|}
\hline & IUE class & & ber of stars & No. & pectra used \\
\hline ID & Description & All & in $\mathrm{GALEX}^{a}$ & SW & LW \\
\hline 10 & WC & 22 & 0 & 70 & 57 \\
\hline 11 & WN & 38 & 0 & 165 & 49 \\
\hline 12 & Main Sequence O & 106 & 1 & 150 & 119 \\
\hline 13 & Supergiant $\mathrm{O}$ & 38 & 0 & 58 & 27 \\
\hline 14 & $\mathrm{Oe}$ & 4 & 0 & 6 & 2 \\
\hline 15 & Of & 10 & 0 & 19 & 5 \\
\hline 16 & SD O & 68 & 29 & 176 & 457 \\
\hline 17 & WD O & 15 & 5 & 20 & 62 \\
\hline 20 & B0-B2 V-IV & 142 & 5 & 293 & 293 \\
\hline 21 & B3-B5 V-IV & 68 & 1 & 87 & 110 \\
\hline 22 & B6-B9.5 V-IV & 138 & 7 & 217 & 136 \\
\hline 23 & B0-B2 III-I & 111 & 2 & 169 & 64 \\
\hline 24 & B3-B5 III-I & 25 & 1 & 48 & 24 \\
\hline 25 & B6-B9.5 III-I & 57 & 3 & 85 & 42 \\
\hline 26 & $\mathrm{Be}$ & 28 & 1 & 67 & 92 \\
\hline 27 & $\mathrm{Bp}$ & 36 & 2 & 63 & 57 \\
\hline 28 & sd B & 36 & 15 & 39 & 27 \\
\hline 29 & WDB & 13 & 7 & 46 & 32 \\
\hline 30 & A0-A3 V-IV & 111 & 5 & 150 & 117 \\
\hline 31 & A4-A9 V-IV & 28 & 2 & 32 & 21 \\
\hline 32 & A0-A3 III-I & 25 & 2 & 31 & 17 \\
\hline 33 & A4-A9 III-I & 17 & 2 & 20 & 8 \\
\hline 34 & $\mathrm{Ae}$ & 4 & 1 & 55 & 4 \\
\hline 35 & Am & 13 & 1 & 16 & 12 \\
\hline 36 & Ap & 20 & 1 & 70 & 87 \\
\hline 37 & WDA & 73 & 26 & 101 & 102 \\
\hline 38 & Horizontal Branch Stars & 26 & 6 & 33 & 18 \\
\hline 40 & F0-F2 & 39 & 3 & 41 & 51 \\
\hline 41 & F3-F9 & 52 & 8 & 60 & 47 \\
\hline 42 & $\mathrm{Fp}$ & 1 & 1 & 1 & 1 \\
\hline 44 & G IV-V & 74 & 20 & 210 & 106 \\
\hline 45 & G III-I & 40 & 7 & 66 & 37 \\
\hline 46 & K V-IV & 50 & 14 & 323 & 112 \\
\hline 47 & K III-I & 35 & 8 & 61 & 16 \\
\hline 48 & M V-IV & 22 & 2 & 106 & 52 \\
\hline 49 & M III-I & 15 & 5 & 35 & 16 \\
\hline 50 & $\mathrm{R}, \mathrm{N}$ or $\mathrm{S}$ Types & 7 & 4 & 9 & 6 \\
\hline 51 & Long Period Variable Stars & 1 & 0 & 1 & 3 \\
\hline 52 & Irregular Variables & 10 & 0 & 18 & 15 \\
\hline 53 & Regular Variables & 30 & 10 & 24 & 104 \\
\hline 54 & Dwarf Novae & 33 & 17 & 62 & 61 \\
\hline 55 & Classical Novae & 21 & 3 & 39 & 51 \\
\hline 58 & T Tauri & 20 & 5 & 30 & 96 \\
\hline
\end{tabular}

Notes. ${ }^{(a)}$ Identified by cross correlation with the GALEX AIS GR5 catalogue. A search radius of 3 arcsec is used. 


\section{References}

Bianchi, L. 2014, Ap\&SS, 304, 103

Bianchi, L., Herald, J., Efremova, B., et al. 2011, Ap\&SS, 335, 161

Boggess, A., Carr, F. A., Fischel, D., et al. 1978, Nature, 275, 372

Bohlin, R. C., Holm, A. V., Savage, D. V., et al. 1980, A\&A, 85, 1

Camarota, L., \& Holberg, J. B. 2014, MNRAS, 438, 3111

Martin, D. C., Fanson, J., Schiminovich, D., et al. 2005, ApJ, 619, L1

Morrissey, P., Schiminovich, D., Barlow, T. A., et al. 2005, ApJ, 619, L7

Morrissey, P., Conrow, T., Barlow, T. A., et al. 2007, ApJS, 173, 682

Rodríguez-Pascual, P., Gonzalez-Riestra, R., Schartel, N., et al. 1999, A\&AS, 139,183

\section{Appendix A: Catalogue excerpt}

An excerpt (first ten entries) of the catalogue is shown in Table A.1 (see Sect. 6, for a detailed description of the fields). The full catalogue is available at the CDS.

\section{Appendix B: FUV magnitude - variable stars}

An excerpt (first ten entries) of the list of FUV magnitudes for variable stars is shown in Table B.1 (see Sect. 6 for a description of the fields). The full table is available at the CDS.

Table A.1. Catalogue layout (first ten entries).

\begin{tabular}{|c|c|c|c|c|c|c|c|c|c|c|}
\hline Object & $\begin{array}{c}\text { RA(2000) } \\
\text { (hh:mm:ss.ss) }\end{array}$ & $\begin{array}{c}\operatorname{Dec}(2000) \\
( \pm d d: m m: s s . s s)\end{array}$ & NobsSWP & $\begin{array}{c}\text { FUV } \\
\text { (ABmag) }\end{array}$ & $\begin{array}{l}+\mathrm{eFUV} \\
\text { (ABmag) }\end{array}$ & $\begin{array}{l}\text {-eFUV } \\
\text { (ABmag) }\end{array}$ & NobsLW & $\begin{array}{c}\text { NUV } \\
\text { (ABmag) }\end{array}$ & $\begin{array}{l}+ \text { eNUV } \\
\text { (ABmag) }\end{array}$ & $\begin{array}{l}\text {-eNUV } \\
\text { (ABmag) }\end{array}$ \\
\hline CD-40 15307 & 00:00:20.14 & $-39: 23: 55.24$ & 1 & 10.143 & 0.003 & -0.003 & 1 & 10.165 & 0.004 & -0.004 \\
\hline WD $2357+296$ & 00:00:7.25 & $+29: 57: 0.31$ & 2 & 13.062 & 0.008 & -0.008 & 0 & NA & NA & NA \\
\hline HD 225094 & 00:03:25.71 & $+63: 38: 25.88$ & 3 & 8.811 & 0.002 & -0.002 & 1 & 8.639 & 0.003 & -0.003 \\
\hline HD 225132 & 00:03:44.39 & $-17: 20: 9.57$ & 6 & 6.422 & 0.001 & -0.001 & 1 & 6.082 & 0.002 & -0.002 \\
\hline HD 108 & 00:06:3.39 & $+63: 40: 46.76$ & 1 & 8.363 & 0.003 & -0.003 & 0 & NA & NA & NA \\
\hline HD 186 & 00:06:47.96 & $+44: 36: 46.2$ & 1 & 9.072 & 0.003 & -0.003 & 1 & 9.213 & 0.004 & -0.004 \\
\hline $\mathrm{BD}+592829$ & 00:06:48.3 & $+60: 36: 0.83$ & 1 & 11.846 & 0.003 & -0.003 & 0 & NA & NA & NA \\
\hline PG 0004+133 & 00:07:33.78 & $+13: 35: 57.66$ & 1 & 13.036 & 0.005 & -0.005 & 1 & 13.276 & 0.007 & -0.007 \\
\hline WD $0005+511$ & 00:08:18.17 & $+51: 23: 16.6$ & 1 & 11.092 & 0.003 & -0.003 & 0 & NA & NA & NA \\
\hline HD 358 & 00:08:23.26 & $+29: 05: 25.55$ & 1 & 3.193 & 0.003 & -0.003 & 0 & NA & NA & NA \\
\hline
\end{tabular}

Table B.1. FUV magnitude for variable stars layout (first ten entries).

\begin{tabular}{cccccccc}
\hline \hline Object & RA(ICRS) & Dec(ICRS) & Obsdate & Obstime & FUV & +eFUV & -eFUV \\
\hline HD 352 & $00: 08: 12.1$ & $-02: 26: 51.76$ & $1985-08-02$ & $10: 10: 00$ & 15.020 & 0.010 & -0.010 \\
HD 352 & $00: 08: 12.1$ & $-02: 26: 51.76$ & $1981-05-20$ & $02: 02: 03$ & 14.345 & 0.004 & -0.004 \\
HD 352 & $00: 08: 12.1$ & $-02: 26: 51.76$ & $1984-06-14$ & $02: 06: 56$ & 15.186 & 0.008 & -0.008 \\
HD 5394 & $00: 56: 42.53$ & $+60: 43: 0.27$ & $1982-01-28$ & $00: 37: 38$ & 6.571 & 0.023 & -0.023 \\
HD 5394 & $00: 56: 42.53$ & $+60: 43: 0.27$ & $1988-07-10$ & $13: 18: 22$ & 1.899 & 0.002 & -0.002 \\
HD 5394 & $00: 56: 42.53$ & $+60: 43: 0.27$ & $1988-07-10$ & $14: 46: 38$ & 1.843 & 0.002 & -0.002 \\
HD 5679 & $01: 02: 18.45$ & $+81: 52: 32.08$ & $1978-05-15$ & $17: 07: 54$ & 9.457 & 0.003 & -0.003 \\
HD 5679 & $01: 02: 18.45$ & $+81: 52: 32.08$ & $1981-08-04$ & $08: 17: 42$ & 15.110 & 0.028 & -0.027 \\
V* RX And & $01: 04: 35.54$ & $+41: 17: 57.8$ & $1980-02-09$ & $04: 05: 26$ & 14.019 & 0.007 & -0.007 \\
V* RX And & $01: 04: 35.54$ & $+41: 17: 57.8$ & $1980-02-28$ & $03: 50: 44$ & 13.895 & 0.007 & -0.007 \\
\hline
\end{tabular}

